\title{
Realization of a Field Emission Gun with Advanced Köhler Illumination
}

\author{
Gerd Benner, Günter Lang, Alexander Orchowski, Wolf-Dieter Rau *, Max Haider \# \\ * LEO Elektronenmikroskopie GmbH, D-73446 Oberkochen, Germany \\ ${ }^{\#}$ CEOS GmbH, D-69126 Heidelberg, Englerstraße 28, Germany
}

We present an evaluation of the performance of a Field Emission Gun (FEG) in combination with the advanced features of a Köhler illumination system. Specifically we address the superior potential of such an illumination system for advanced material characterization in HRTEM, STEM and nanoanalytic applications.

The investigation of structure, composition and bonding of crystals and buried interfaces is highly important for the understanding of the properties of modern materials. The atomistic structure of specific interfaces is obtained by high resolution TEM, whereas analytical electron microscopic investigations deliver information on the chemical composition, often required to be locally resolved in the sub-nm range.

To analyse these dimensions for example by EELS, a small probe with high beam current is required. For STEM also a high brightness of the source is needed in order to achieve a Z-contrast resolution in the range $0.2 \mathrm{~nm}$. On the other hand for imaging the specimen structure, an isotropic illumination (over a wide range) is essential to avoid artefacts by tilted illumination. Furthermore, the illumination system should allow to select different illumination conditions, e. g. to switch from $1 \mathrm{~nm}$ spot to a large (parallel) illuminated area without any need of realignment. All these requirements can be fulfilled perfectly by a Field Emission Gun with Schottky Emitter and a Köhler illumination system [1]. A first prototype of this illumination system was realized in a $200 \mathrm{kV}$ TEM with $90^{\circ}$ Omega in-column imaging filter (SESAMe II) [2].

Fig. 1 shows the set-up and the principal ray path. The FEG consists of an emitter assembly followed by an electrostatic Einzel-lens (gun lens) and a 6-stage accelerator. The gun lens is optimized regarding axial aberration and is used to provide a stable Cross-Over (CO) position when varying the extraction voltage. An energy width (full width half maximum FWHM) of the transmitted electron beam between 0.85 and $0.62 \mathrm{eV}$ is achieved (Fig. 2). The gun assembly is prepared for integration of a dispersion-free monochromator of electrostatic $\Omega$-type [3], enabling energy widths below $0.2 \mathrm{eV}$, thus allowing for the most sophisticated energy filtering applications.

In Spot mode the gun $\mathrm{CO}$ is imaged by the condenser zoom system into the object plane of the objective prefield lens (broken line), resulting in a strongly demagnified spot on the specimen. Spot size can be varied by different excitations of the condenser lens system. In TEM Mode the condenser zoom system provides a $\mathrm{CO}$ image with variable size in the front focal plane of the objective prefield lens (solid line). Keeping the CO-image at a constant plane for all brightness steps results in an axial parallel illumination over a large area.

Fig. 3 shows the profile of the magnified spot imaged on a SS-CCD camera. The measured FWHM of the spot profile is $0.32 \mathrm{~nm}$. Due to the high brightness of the FEG $\left(5 \cdot 10^{8} \mathrm{~A} / \mathrm{cm}^{2} \mathrm{sr}\right)$ a probe current of $600 \mathrm{pA}$ in a spot size of $0.5 \mathrm{~nm}$ is achieved. In order to demonstrate the uniformity of the illumination, an image of carbon foil was recorded by a $2 \mathrm{k}$ SS-CCD camera at a microscope magnification of $M=50 \mathrm{k}$ (Fig. 4a). Numerical diffractograms calculated at center and corners are shown in Fig. 4b. The similar shape and diameter of the Thon rings indicate, that there is no change in focus and tilt over this large field of view (FOV). 


\section{References}

[1] Benner, G. and Probst, W., Journal of Microscopy, Vol.174, (1994), 133

[2] Lang, G. and Benner, G., (2002) Microsc. Microanal., this volume

[3] Uhlemann, S. and Haider, M., (2002) Microsc. Microanal., this volume

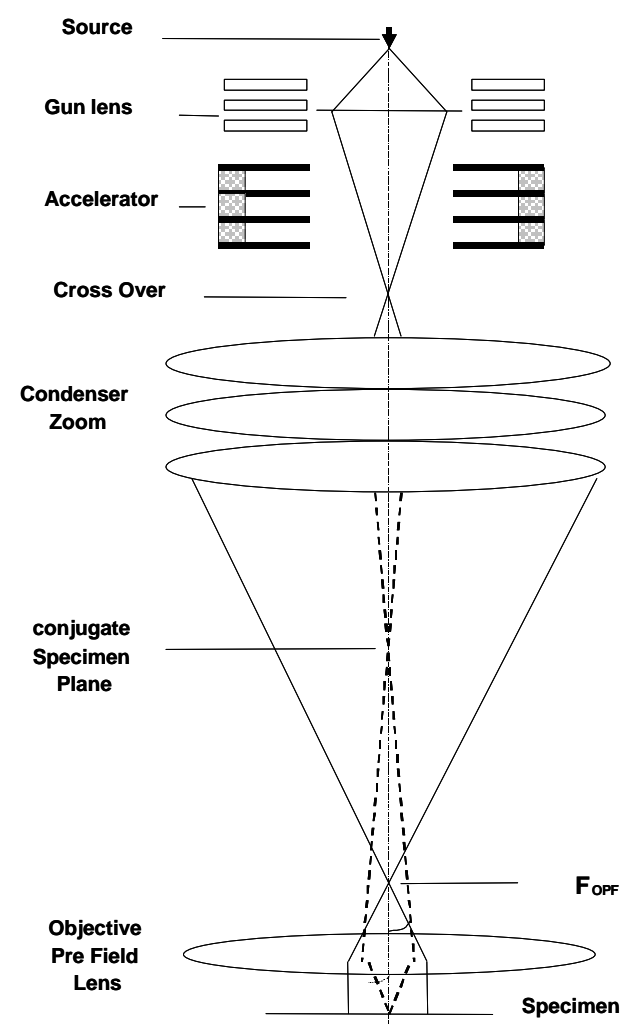

Fig. 1: Principal set-up and ray-path

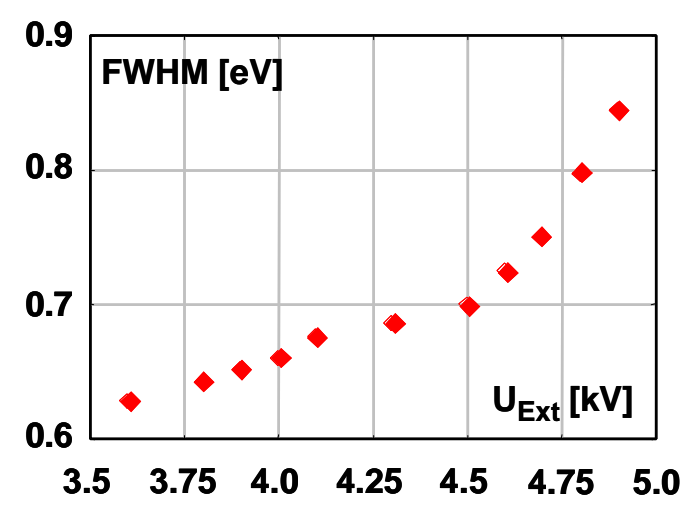

Fig. 2: Energy-width (FWHM) of the electron beam as function of applied extraction voltage $\mathrm{U}_{\mathrm{Ext}}$

Corresponding author: Benner@leo.de

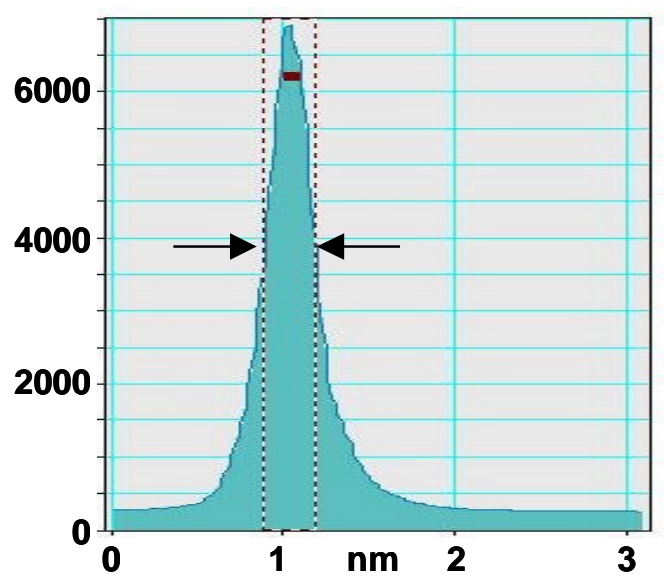

Fig. 3: Spotprofile (FWHM $0.32 \mathrm{~nm}$ )

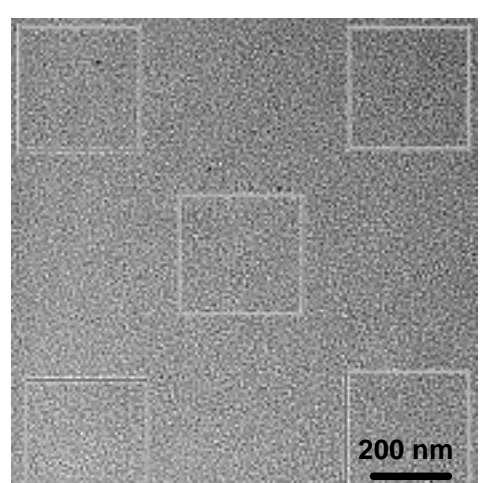

Fig. 4:

CTF of carbon foil at the center and the corners of $1,2 \mu \mathrm{m}$ FOV
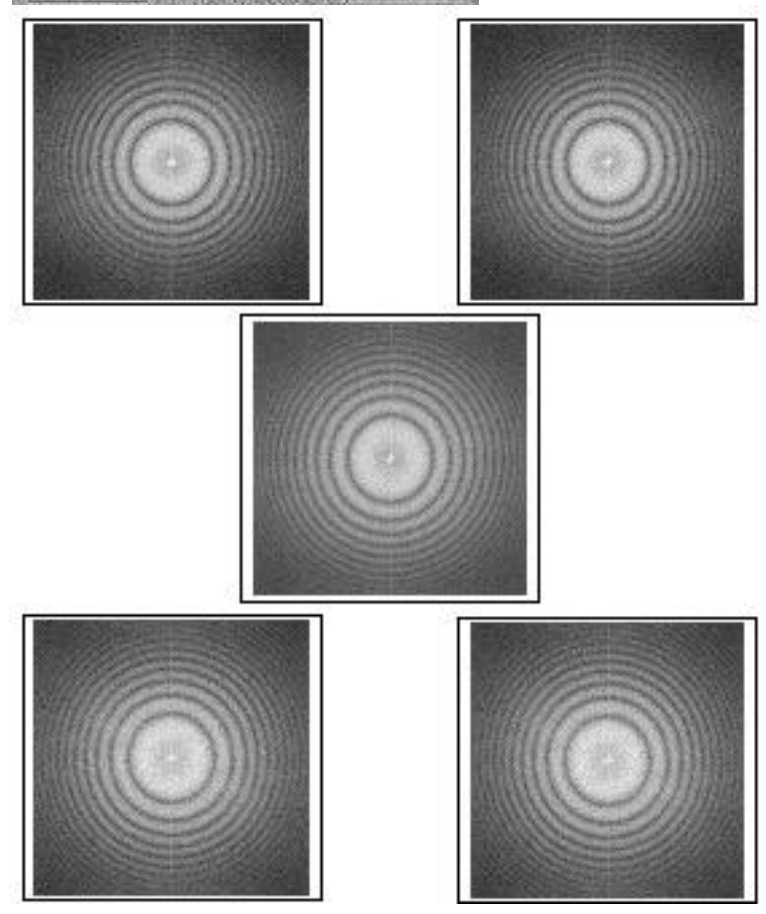